\title{
Quantum Tunneling Detection of Two-Photon and Two-Electron Processes
}

\author{
J. Tobiska, ${ }^{1}$ J. Danon, ${ }^{1}$ I. Snyman, ${ }^{2}$ and Yu. V. Nazarov ${ }^{1}$ \\ ${ }^{1}$ Kavli Institute of NanoScience, Delft University of Technology, 2628 CJ Delft, The Netherlands \\ ${ }^{2}$ Instituut-Lorentz, Universiteit Leiden, P.O. Box 9506, 2300 RA Leiden, The Netherlands
}

(Received 24 June 2005; published 7 March 2006)

\begin{abstract}
We analyze the operation of a quantum tunneling detector coupled to a coherent conductor. We demonstrate that, in a certain energy range, the output of the detector is determined by two-photon processes, two-interacting-electron processes, and the interference of the two. We show how the individual contributions of these processes can be resolved in experiments.
\end{abstract}

DOI: 10.1103/PhysRevLett.96.096801

The quantum nature of electron transfer in coherent conductors is seldom explicitly manifested in averaged current-voltage curves. To reveal it, one should measure current noise and/or higher-order correlations of current comprising full counting statistics which arise from the transfer [1]. Such measurements not only reveal the discrete nature of the charges transferred, they also quantify quantum many-body effects in electron transport and may be used for the detection of pairwise entanglement of transferred particles [2-5]. If the noise is measured at frequencies in the quantum range, $\hbar \omega \gg k_{\mathrm{B}} T$, the measurement amounts to the detection of photons produced by the current fluctuations. This aspect is important in view of attempts to transfer quantum information from electrons to photons and back [6].

It was demonstrated in Ref. [7] that one needs a quantum detector to measure quantum noise. Indeed, any classical measurement of a fluctuating quantity would give a noise spectrum symmetric in frequency, $S(\omega)=S(-\omega)$. A quantum tunneling detector is generally a quantum twolevel system with a level separation $\varepsilon>0$. The results of detection are two transition rates: $\Gamma_{\text {up }}$ from the lower to the higher level and $\Gamma_{\text {down }}$ for the reverse direction. The most probable transitions are accompanied by either absorption or emission of a photon of matching energy $\hbar \omega=\varepsilon$. One can define the noise spectrum in such a way that it is proportional to the transition rates $S( \pm \varepsilon / \hbar) \propto \Gamma_{\text {up,down }}(\varepsilon)$. Differences between $\Gamma_{\text {up,down }}$ thus manifest the quantum nature of noise. If the source of noise is a coherent conductor biased by a voltage $V$, detector signals in the range $\varepsilon<e V$ are readily interpreted in terms of single-electron transfers through the conductor. The maximum energy gain available for electrons in the course of such transfer is $e V$. Consequently, this value also limits the energy of the emitted photon. The previous research has not addressed the energy range $\varepsilon>e V$. It remained unclear if the detector detects anything and - if positive - what it detects.

A first proposal for the experimental realization of a quantum tunneling detector included transitions between two localized electron states in a double quantum dot [8]. However, it does not matter much if the tunneling occurs
PACS numbers: 73.40.Gk, 05.40.- a, 72.70.+m, 73.23.-b between localized or delocalized electron states and if all tunnel events are accompanied by the same energy transfer $\varepsilon$. In most practical cases, the energy dependence of the rates $\Gamma_{\text {up,down }}$ can be readily extracted from the measurement results. This is why quantum tunneling detection has been experimentally realized in a superconducting tunnel junction [9] and in a single quantum dot [10].

In this Letter, we study quantum tunneling detection in the range $e V<\varepsilon<2 e V$ assuming $\varepsilon, e V \gg k_{\mathrm{B}} T$. The motivation is that for these energies the detector is not sensitive to single-electron one-photon processes described above and its output - the transition rate $\Gamma_{\text {up }}$-is determined by much more interesting two-particle processes. It is clear from plain energy considerations that transitions may originate from two-photon processes. Such twophoton absorption can occur given any nonequilibrium photon distribution bounded by $e V$, not necessarily produced by a coherent conductor. Less obvious and specific for a coherent conductor is a cooperative two-electron process. Indeed, if two electrons team up in crossing the conductor, they can emit a single photon with an energy up to $2 \mathrm{eV}$. Essential for this cooperation are electron-electron interactions. It is known [11] that the most important electron-electron interaction in this energy range is due to the electromagnetic environment of the conductor, the same environment in which the nonequilibrium photons dwell.

We quantify the signals due to two-photon and twoelectron events and find them to be of the same order of magnitude. We also show that part of the signal is due to quantum interference of these two processes: one-and-ahalf-photon absorption events. We demonstrate how different contributions can be separated in experiments, thereby facilitating the direct observation of two-particle processes in the context of quantum transport.

We concentrate on a model circuit consisting of four elements as given in Fig. 1. A voltage biased coherent contact characterized by a set of transmission eigenvalues $\left\{T_{n}\right\}$ is embedded in an electromagnetic environment with impedance $Z_{\omega}$. The environment transforms the current fluctuations in the conductor to voltage fluctuations in 


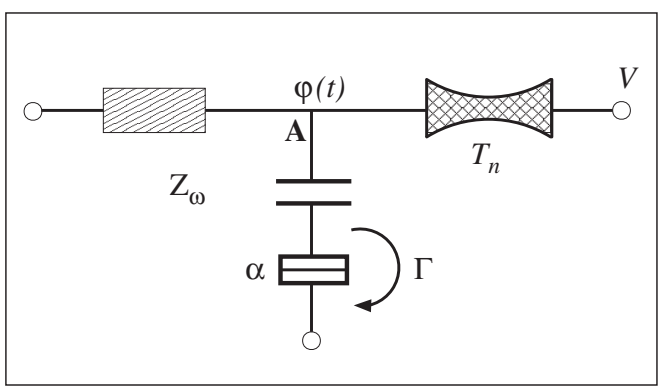

FIG. 1. Model circuit.

node $A$ which are conveniently expressed in terms of a phase $\varphi=\frac{e}{\hbar} \int d t V(t)$. The most general model including the detector and coherent contact would be a four-pole circuit studied in Ref. [12] that couples two poles of the detector with two poles of the contact. Here we concentrate on the experimentally relevant case of capacitive coupling. Owing to voltage division between two capacitors, the detector senses a fraction $0<\alpha<1$ of the voltage fluctuations in node $A$. We will see that changing the "visibility" parameter $\alpha$ enables the separation of two-electron and two-photon processes in experiments. The relevant impedance is made up of an environmental impedance combined with that of two capacitors and that of the coherent contact. We measure this impedance $z_{\omega}$ in units of $R_{K} \equiv 2 \pi \hbar / e^{2}$ and assume the low-impedance limit $z_{\omega} \ll 1$; this provides us with a physically justified small parameter.

The detector consists of two localized charge states connected by a tunnel amplitude $\mathcal{T}$. In the presence of voltage fluctuations in the node $A$, the amplitude is modified as follows: $\mathcal{T}(t) \rightarrow \mathcal{T} e^{i \alpha \varphi(t)}$. In perturbation theory, the inelastic tunneling rate between two states separated by $\varepsilon$ is given by correlators of $\alpha \varphi(t)$ [11]

$$
\Gamma(\varepsilon)=\frac{|\mathcal{T}|^{2}}{2 \pi \hbar^{2}} \int d t\left\langle e^{i \alpha \varphi(t)} e^{-i \alpha \varphi(0)}\right\rangle e^{(i / \hbar) \varepsilon t} .
$$

The rate $\Gamma(\varepsilon)$ is therefore the Fourier transform of the correlation function $\left\langle e^{i \alpha \varphi(t)} e^{-i \alpha \varphi(0)}\right\rangle, \alpha \varphi(t)$ being the phase fluctuations over the detector. From now on, we take $\hbar=e=k_{\mathrm{B}}=1$.

Equation (1) tells us that the inelastic tunneling rates in the detector are completely determined by the voltage fluctuations over the junction. Therefore, measuring the inelastic current through the dots, we are sensitive to the noise spectrum of the environment.

To evaluate $\left\langle e^{i \alpha \varphi(t)} e^{-i \alpha \varphi(0)}\right\rangle$, we construct a path integral representation of this quantity using a nonequilibrium Keldysh technique [13] for quantum circuits [14]

$$
\begin{aligned}
\left\langle e^{i \alpha \varphi(t)} e^{-i \alpha \varphi(0)}\right\rangle= & \int \mathcal{D}[\phi] \exp \left\{-i S_{\mathrm{env}}[\phi]-i S_{\mathrm{cond}}[\phi]\right. \\
& \left.+i \alpha\left[-\varphi^{+}(0)+\varphi^{-}(t)\right]\right\} .
\end{aligned}
$$

The integration goes over the time-dependent fluctuating fields $\varphi^{ \pm}(t)$ in node $A, \pm$ corresponding to the forward (backward) part of the Keldysh contour. $S_{\text {env }}$ and $S_{\text {cond }}$ are the contributions to the Keldysh action originating from the environment and the coherent conductor, respectively.

Since the environment is linear, its action is quadratic in the fields and at zero temperature reads (cf. [15])

$$
S_{\mathrm{env}}=\int d \omega \phi_{-\omega}^{T} A(\omega) \phi_{\omega},
$$

with

$$
A(\omega)=-\frac{i}{2}\left(\begin{array}{cc}
0 & -\frac{\omega}{z_{-\omega}} \\
\frac{\omega}{z_{\omega}} & |\omega| \operatorname{Re}\left\{\frac{1}{z_{\omega}}\right\}
\end{array}\right),
$$

$z_{\omega}$ being the corresponding impedance. We use Fourier transformed fields $\phi_{\omega}=\left(\phi_{\omega}, \chi_{\omega}\right)^{T}$ defined with $\varphi^{ \pm}=$ $\phi \pm \frac{1}{2} \chi$.

All nonquadratic contributions to the action originate from the coherent conductor. The action $S_{\text {cond }}$ can be expressed in terms of Keldysh Green functions $\breve{G}_{L, R}$ of electrons in the reservoirs left and right of the contact [15]

$$
S_{\text {cond }}=\frac{i}{2} \sum_{n} \operatorname{Tr} \ln \left[1+\frac{T_{n}}{4}\left(\left\{\check{G}_{L}(\phi), \check{G}_{R}\right\}-2\right)\right] .
$$

The fields $\phi$ enter in this action via the gauge transform of $\breve{G}_{L}[15]$.

To comprehend the physics involved, let us first disregard any nonquadratic parts and take only the quadratic part of $S_{\text {cond. }}$. In this case, the path integral is Gaussian and can be evaluated exactly. We recover the well known result from $P(E)$ theory (cf. [8,11]): $\left\langle e^{i \alpha \varphi(t)} e^{-i \alpha \varphi(0)}\right\rangle=\exp [J(t)]$, with

$$
J(t)=\langle\alpha \varphi(t) \alpha \varphi(0)\rangle=\alpha^{2} \int d \omega \frac{\left|z_{\omega}\right|^{2}}{\omega^{2}} K(\omega)\left[e^{-i \omega t}-1\right] .
$$

The impedance includes the dimensionless conductance $g_{c} \equiv \sum_{n} T_{n}$ of the contact.

In the limit of $T=0$, we find in agreement with results of Refs. [7,8]

$$
\begin{aligned}
K(\omega)= & g_{c}\{F D(\omega+V)+(2-2 F) D(\omega) \\
& +F D(\omega-V)\}+2 \operatorname{Re}\left\{\frac{1}{z_{\omega}}\right\} D(\omega),
\end{aligned}
$$

with $D(\omega) \equiv-\omega \theta(-\omega)$ and the Fano factor $F \equiv$ $\sum_{n} T_{n}\left(1-T_{n}\right) / \sum_{n} T_{n}$. The first term in $K(\omega)\left(\propto g_{c}\right)$ represents the nonequilibrium current noise spectrum of the coherent contact that vanishes for $\omega>V$. In physical terms, this means that the highest energy $\omega$ an electron can emit traversing the conductor is exactly $V$. The second part represents the spectrum of the environment. It is zero for $\omega>0$, since the environment can absorb energy only at $T=0$.

The time-dependent part of $J(t)$ is the Fourier transform of $K(\omega)\left|z_{\omega}\right|^{2} / \omega^{2}$ and $\Gamma(\varepsilon)$ is, in turn, the Fourier transform of $\exp [J(t)]$. If we expand $\exp [J(t)]$ in terms of $J(t)$, the $n$th 
term presents the contribution of a process involving absorption of $n$ photons in the detector. Such an $n$-photon process dominates in the interval $(n-1) V<\varepsilon<n V$ and its contribution is proportional to $\alpha^{2 n}$.

The one-photon contribution gives $\Gamma_{\text {up }}^{(1)} / \Gamma_{\text {down }} \simeq z g_{c} F$. Each extra photon brings in a small factor, such that $\Gamma^{(n+1)} / \Gamma^{(n)} \simeq \alpha^{2} z^{2} g_{c} F$. This is seen as a staircase in the log plot presented in Fig. 2.

What we did was wrong, since we did not take into account the nonquadratic terms in the action. These describe more interesting many-electron processes and areas we show below - of the same order of magnitude. Since the path integral in (2) cannot be evaluated in general, we proceed by perturbative expansion.

Indeed, since $|z| \ll 1$, the Gaussian part of the action, being proportional to $z^{-1}$, suppresses fluctuations in the path integral, and we can treat the remaining part perturbatively. First we expand $-i S_{\text {cond }}[\phi]$ around $\phi=0$. As mentioned previously [see the discussion below Eq. (5)], the first- and second-order terms just renormalize the impedance. The exponential of the remaining higher-order terms is then again expanded in $\phi$ around $\phi=0$. This expansion may be represented in terms of diagrams such as those in Fig. 3. Figure 3(a) represents a high-order term, from which the general structure becomes clear: Diagrams consist of lines, polygons, and external vertices. The expansion contains not only connected diagrams but all disconnected diagrams as well. A polygon with $n$ vertices is associated with the symmetrized $n$th order coefficient in the Taylor expansion of $-i S_{\text {cond }}[\phi]$. Each polygon contributes a factor $g_{c}$. Lines represent propagators of $\phi$ corresponding to the Gaussian action with renormalized impedance. They are of order $z$, making $n$-line diagrams $z^{n}$ in leading order. External vertices (indicated by dots in the figure) are associated with the time-dependent linear term $i \alpha\left[-\varphi^{+}(0)+\varphi^{-}(t)\right]$ in Eq. (2). Thus, a diagram with $s$ external vertices gives a correction proportional to $\alpha^{s}$.

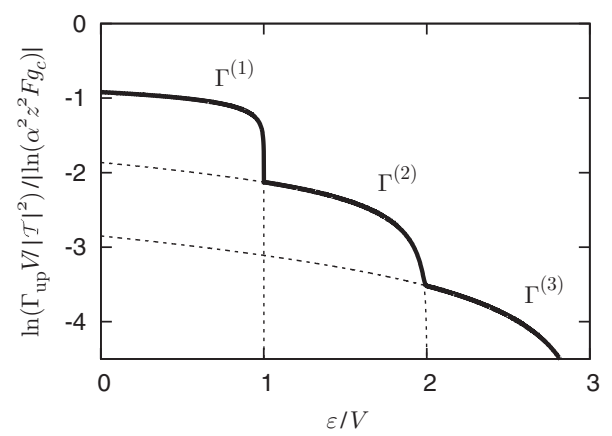

FIG. 2. n-photon contributions (dashed lines) to the detector output. Each contribution dominates in the energy interval $(n-$ 1) $V<\varepsilon<n V$. Subsequent contributions are suppressed by a factor $\approx \alpha^{2} z^{2} g_{c} F$. This is seen as a staircase structure in the log plot. The sum of all contributions is given by the solid line. To produce this plot, we took $z_{\omega} / \omega=z / V, z=0.01, g_{c} F=1.75$, $\alpha=1$.
Furthermore, diagrams without external vertices are timeindependent and, according to Eq. (1), contribute only to elastic tunneling processes. Figures 3(b)-3(f) represent some of the lowest-order terms in the expansion.

We consider transitions in the detector where energies between $V$ and $2 V$ are absorbed. In this interval, the detector output is given by Figs. 3(b) $-3(d)$, which are proportional to $\alpha^{4}, \alpha^{3}$, and $\alpha^{2}$, respectively.

From the results presented in Fig. 2, we have learned that $n$-photon processes come with a coefficient $\alpha^{2 n}$. Hence, the $\alpha^{3}$ contribution is not readily expected: It seems to signal a process with one-and-a-half photons absorbed. We disregard Fig. 3(e), which contributes only to elastic processes. In the energy interval considered, the combined $z^{3}$ contribution of the included diagrams is zero, and we obtain a tunneling rate that goes as $g_{c}^{2} z^{4}$. Since a diagram like Fig. 3(f) has four lines, it could potentially contribute to the current with the same order in $z$. However, its contribution can only be proportional to $g_{c}$ and is disregarded.

The expansion of $S_{\text {cond }}$ up to fourth-order terms and subsequent evaluation of the diagrams is straightforward but requires rather involved and lengthy calculations. Fortunately, in the interval of interest the three contributions can be combined in a compact expression

$$
\begin{aligned}
\Gamma_{\text {up }}= & 2|\mathcal{T}|^{2} g_{c}^{2} F^{2} \int_{\varepsilon-V}^{V} d \omega(V-\omega)(\varepsilon-V-\omega) \frac{\left|z_{\omega}\right|^{2}}{\omega^{2}} \\
& \times\left|\frac{\alpha^{2}}{2} \frac{z_{\varepsilon-\omega}}{\varepsilon-\omega}+\alpha \frac{z_{\varepsilon}}{\varepsilon}\right|^{2},
\end{aligned}
$$

which is the main result of our work. The rate is proportional to the square of the zero-frequency current noise $S_{\text {cond }}(0)=\frac{2}{\pi} g_{c} F$.

The part proportional to $\alpha^{4}$ [Fig. 3(b)] represents a twophoton process originating from the quadratic part of $S_{\text {cond }}$ and was already present in Fig. 2. We have thus shown that there are contributions of the same order resulting from nonlinearities in the conductor. The $\alpha^{2}$ term [Fig. 3(d)] is the result of the two-electron and one-photon process expected from general reasoning presented in the introduction. We see that the $\alpha^{3}$ term comes from the cross term in

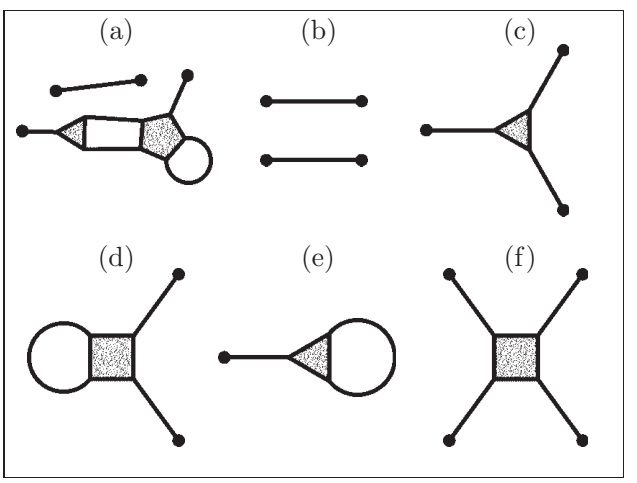

FIG. 3. Typical Feynman diagrams appearing in the expansion of the path integral representation of the tunneling rate. 


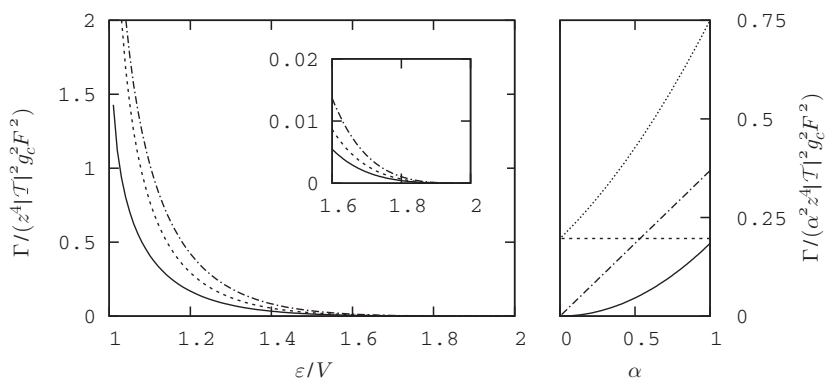

FIG. 4. Left: Contributions to the detector output due to twophoton (solid line), two-electron (dashed line) processes, and their interference (dashed-dotted line) at $\alpha=0.8$ versus detector level splitting. The inset presents a zoom at $\varepsilon \rightarrow 2 V$. Right: Different dependence on the coupling strength $\alpha$ enables experimental identification of these three contributions. The contributions and their sum (dotted line) are plotted for $\varepsilon=1.3 \mathrm{~V}$.

the modulus square. This unambiguously identifies Fig. 3(c) as the result of quantum interference of the twoelectron process and the two-photon processes - an interpretation that was not obvious from the beginning.

To understand this interference, we note that the photon modes involved are delocalized across the whole circuit. A photon in each mode can be absorbed in the detector as well as in the environment or the contact. An elementary process is such that the final state differs from the initial state by two photons absorbed in two given modes. The final state can be reached by two amplitudes: one with both photons absorbed in the detector and one with a photon absorbed in the detector and a photon absorbed in the environment. While the squares of these amplitudes represent the probabilities of two-photon and two-electron processes, respectively, their cross term gives rise to an interference contribution $\propto \alpha^{3}$.

The simplest concrete model is that of a frequencyindependent impedance, $z_{\omega}=z$ at $\omega \simeq V$. The integration in Eq. (7) yields for the three distinct contributions $(\tilde{\varepsilon}=$ $\varepsilon / V, 1<\tilde{\varepsilon}<2$ )

$$
\frac{\Gamma_{i}}{z^{4}|\mathcal{T}|^{2} g_{c}^{2} F^{2}}=\left\{\begin{array}{l}
\alpha^{4}\left[-\frac{2-2 \tilde{\varepsilon}+\tilde{\varepsilon}^{2}}{\tilde{\varepsilon}^{3}} \ln (\tilde{\varepsilon}-1)-\frac{2-\tilde{\varepsilon}}{\tilde{\varepsilon}^{2}}\right] \\
2 \alpha^{3}\left[-\frac{2-2 \tilde{\varepsilon}+\tilde{\varepsilon}^{2}}{\tilde{\varepsilon}^{3}} \ln (\tilde{\varepsilon}-1)-\frac{2-\tilde{\varepsilon}}{\tilde{\varepsilon}^{2}}\right] \\
\alpha^{2}\left[-\frac{2}{\tilde{\varepsilon}} \ln (\tilde{\varepsilon}-1)-\frac{4(2-\tilde{\varepsilon})}{\tilde{\varepsilon}^{2}}\right] .
\end{array}\right.
$$

All contributions scale as $(\varepsilon-2 V)^{3}$ at the two-photon threshold and logarithmically diverge at approaching the one-photon threshold (see Fig. 4). Eventually, this divergence is cut off in the close vicinity of $\varepsilon=e V$ at an energy scale $g z^{2} e V$ where two-particle and one-photon rates become comparable.

Here we have quantified the contributions for a very specific nonlinear quantum noise source: a coherent conductor. However, in the case of any unknown source of this kind, the $\alpha$ dependence of the contributions allows one to separate and identify them experimentally (right pane in
Fig. 4). One would measure the detector output changing the coupling to the detector. Formally, three measurements at three different $\alpha$ are sufficient to determine the relative strength of all three contributions. In any case, in the limit of small coupling $\alpha \rightarrow 0$ the detector output is dominated by two-electron events. Further characterization may be achieved by engineering of a frequency-dependent impedance. For instance, setting $z(\omega=\epsilon)$ to 0 eliminates both interference and two-electron contributions.

To conclude, we have shown that the quantum tunneling detector in the energy interval specified is selectively sensitive to two-particle processes. The detector output is generally determined by three contributions: two-photon processes, two-electron processes, and the interference of the two. These three sources can be distinguished experimentally by measuring at different couplings $\alpha$ to the detector. Our results thus facilitate the direct observation of many-particle events in the context of quantum transport.

This work was supported by the Dutch Foundation for Fundamental Research on Matter (FOM).

[1] Y. M. Blanter and M. Büttiker, Phys. Rep. 336, 1 (2000).

[2] NATO Advanced Research Workshop on Quantum Noise in Mesoscopic Physics, Quantum Noise in Mesoscopic Physics, edited by Y. V. Nazarov, NATO Science Series II Vol. 97 (Kluwer Academic, Delft, The Netherlands, 2003).

[3] A. Di Lorenzo and Y. V. Nazarov, Phys. Rev. Lett. 94, 210601 (2005).

[4] C. W. J. Beenakker and M. Kindermann, Phys. Rev. Lett. 92, 056801 (2004).

[5] P. Samuelsson, E. V. Sukhorukov, and M. Büttiker, Phys. Rev. Lett. 92, 026805 (2004).

[6] V. Cerletti, O. Gywat, and D. Loss, Phys. Rev. B 72, 115316 (2005).

[7] U. Gavish, Y. Levinson, and Y. Imry, Phys. Rev. B 62, R10 637 (2000).

[8] R. Aguado and L. P. Kouwenhoven, Phys. Rev. Lett. 84, 1986 (2000).

[9] R. Deblock, E. Onac, L. Gurevich, and L.P. Kouwenhoven, Science 301, 203 (2003).

[10] F. Balestro, E. Onac, U. Hartmann, Y. V. Nazarov, and L. P. Kouwenhoven (unpublished).

[11] G. L. Ingold and Y. V. Nazarov, in Single Charge Tunneling, edited by $\mathrm{H}$. Grabert and $\mathrm{M}$. Devoret (Plenum, New York, 1992), p. 21.

[12] M. Kindermann, Y. V. Nazarov, and C. W. J. Beenakker, Phys. Rev. B 69, 035336 (2004).

[13] J. Rammer and H. Smith, Rev. Mod. Phys. 58, 323 (1986).

[14] M. Kindermann, Y. V. Nazarov, and C. W. J. Beenakker, Phys. Rev. Lett. 90, 246805 (2003).

[15] M. Kindermann and Y. V. Nazarov, Phys. Rev. Lett. 91, 136802 (2003). 